\title{
EHMTI-0039. Different efficacy of acute migraine therapies for migraine with aura versus without aura
}

\author{
J Møller Hansen 1*, PJ Goadsby², A Charles ${ }^{1}$ \\ From 4th European Headache and Migraine Trust International Congress: EHMTIC 2014 \\ Copenhagen, Denmark. 18-21 September 2014
}

\section{Objective}

To determine if acute migraine treatment outcome is different in patients with migraine with aura compared those with migraine without aura.

\section{Methods}

We extracted treatment outcome for migraine with and without aura from large-scale randomized clinical trials of two different drugs with proven efficacy in migraine treatment; sumatriptan and inhaled dihydroergotamine (DHE).

\section{Results}

The pain free rates $2 \mathrm{~h}$ post-dose for sumatriptan 100 mg were significantly higher in patients treating attacks without aura (32\%), compared to the group who treated attacks with aura $(24 \%),(P<0.001)$. For DHE the $2 \mathrm{~h}$ pain free rates did not differ between patients treating attacks without aura (29.4\%) compared to those who treated attacks with aura $(27.2 \% ; \mathrm{P}=0.65)$, see figure. The NNT to achieve $2 \mathrm{~h}$ pain free for sumatriptan was 4.3 for attacks without aura and 6.2 for attacks with aura. For DHE, NNT for $2 \mathrm{~h}$ pain free was 5.8 for attacks without aura and 5.0 for attacks with aura.

\section{Conclusion}

These data indicate that sumatriptan is less effective as acute therapy for migraine attacks with aura compared to attacks without aura. Inhaled DHE, by contrast, had similar efficacy for migraine attacks with and without aura. Different responses of migraine with vs. without aura to acute therapies may provide insight into underlying mechanisms of the disorder. In addition, these different responses may have implications regarding design of

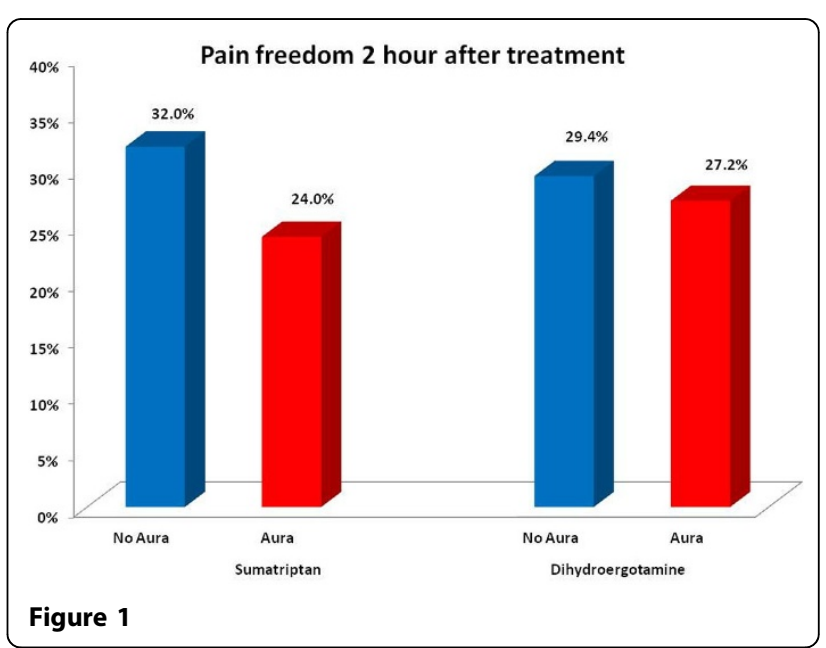

clinical trials, and may influence the choice of acute therapies for different types of migraine attacks.

No conflict of interest.

\section{Authors' details}

'Department of Neurology: Headache Research and Treatment Program, University of California Los Angeles (UCLA), Los Angeles, USA. ²Department of Neurology: Headache Group, University of California San Francisco (UCSF), San Francisco, USA.

Published: 18 September 2014

doi:10.1186/1129-2377-15-S1-G26

Cite this article as: Hansen et al.: EHMTI-0039. Different efficacy of acute migraine therapies for migraine with aura versus without aura. The Journal of Headache and Pain 2014 15(Suppl 1):G26. 